\title{
WATER CYCLE ALGORITHM BASED POWER SYSTEM STABILIZER ROBUST DESIGN FOR POWER SYSTEMS
}

\author{
Navid Ghaffarzadeh
}

\begin{abstract}
Power System Stabilizers (PSSs) are used to enhance damping of power system oscillations. This paper presents a new fast algorithm to develop PSS. According to this regard, Water Cycle Algorithm (WCA) is used to detect optimum Power System Stabilizer parameters. For this purpose, the design problem of PSS is formulated as an optimization problem and WCA by forming three subsidiary objective functions is employed to search for optimal power system stabilizer's parameters to minimize the maximum overshoot and settling time together. In order to show the efficiency of the proposed algorithm, a comparison between WCA and without PSS condition has been done, and the results of proposed algorithm show that it detects optimum parameters in reasonable computation time to increase the power system stability.

K e y w o r d s: power system stabilizer (PSS), water cycle algorithm (WCA), power system stability, optimum parameters
\end{abstract}

\section{INTRODUCTION}

With development of power systems, connecting them to each other is inevitable but it will lead to create oscillations over the frequency range from 0.2 to $3 \mathrm{~Hz}$ in the systems [1]. If these low frequency oscillations are occurred in power systems and adequate damping devices are not provided, this may cause system instability and even loss of synchronism [2-4].

In order to damping oscillations, the generators should be equipped with power system stabilizers (PSSs) that provide supplementary feedback signals to do stabilizing Power systems. Two main approaches have been introduced in the literature that stabilize a power system over a wide range of operation conditions; adaptive control and robust control. Each of these methods has advantages and disadvantages that have been verified in [5]. In the recent years, several conventional techniques such as the eigenvalue assignment, gradient procedure for optimization, mathematical programming, and also the modern control theory, have been proposed in the literature pertaining to design problems of conventional power system stabilizers [2]. In [6] Power system Stabilizer (PSS) design is done with output feedback, but these controllers, need to centralized estimators and controllers. Fuzzy logic methods and artificial neural networks is used to design power system stabilizer (PSS) in [7-9]. Recently, various optimization techniques such as genetic algorithm [10,11], evolutionary programming algorithm [12], steel plating process [4], and particle swarm optimization (PSO) technique $[1,2,4,5,13-15]$ have been used for this purpose, but some of them have limits in optimizing, for example genetic algorithm highly depends on the choice range of each parameter to be optimized [10].

In this paper water cycle algorithm is used to optimal design power system stabilizer (PSS). The idea of this algorithm is received from natural water cycle which is began by initialing of variables as Raindrops, then by forming the river and sea, evaporation is done and cycle is continued again until the best possible answer which is value of sea is fined. One advantage of this algorithm is in raining stage which in the probability of trapping in local maximum or minimum points is lost.

In most works, for designing the PSS, four or five variables is used which reduce efficiency and speed, but in this paper, only three parameters are considered as variables to design PSS, so the efficiency and speed for finding optimal PSS parameters is increased. Finally, this paper is organized as follows.

\section{SYSTEM UNDER STUDY}

\subsection{Power System Model}

The operations of generator, load, controllers and connecting them properly can be modeled with a set of differential- algebraic equations based on Heffron-Philips model. The system under study is shown in Fig. 1 and consisted of a single machine infinite bus system. In [16] model of a synchronous generator has been represented that consists of a winding on the q axis with field circuit. Also in [16] as well as [5] the dynamic model of the single machine connected to an infinite bus system is considered

\footnotetext{
* Faculty of Engineering and Technology, Imam Khomeini International University, Qazvin, Iran, ghaffarzadeh@eng.ikiu.ac.ir
} 


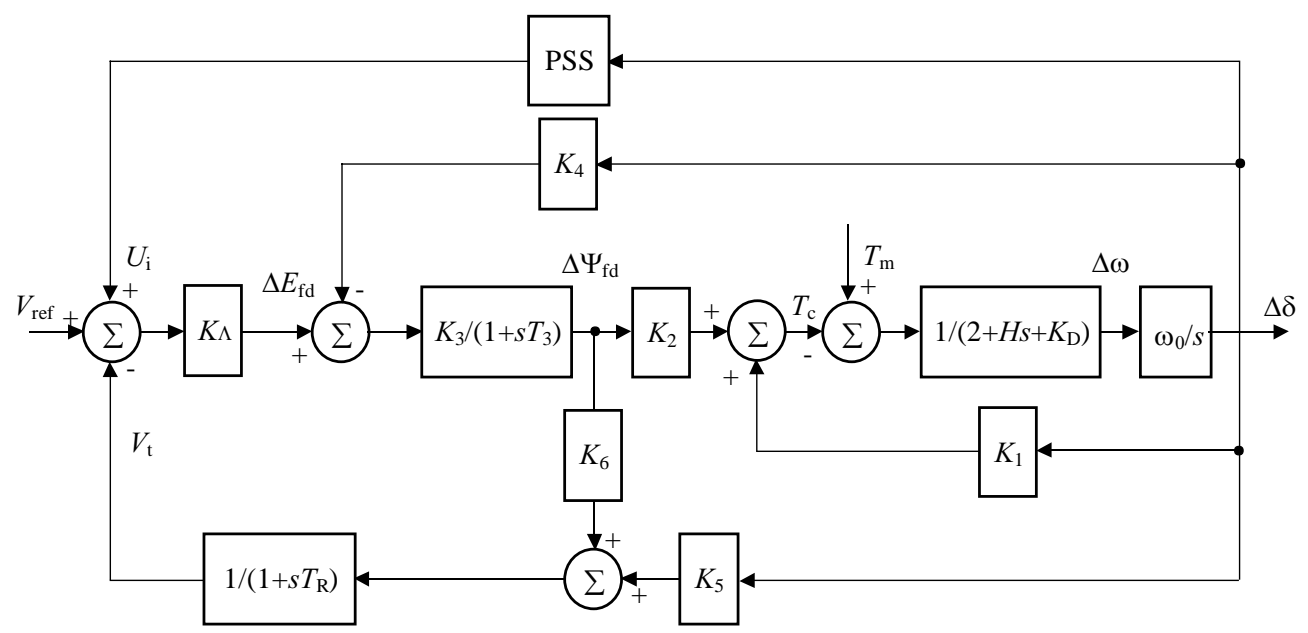

Fig. 1. The system under study

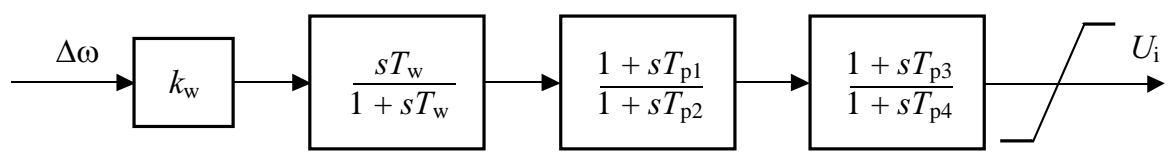

Fig. 2. The structure of power system s tabilizer (PSS)

and its equations can be summarized as

$$
\begin{gathered}
\Delta \dot{\delta}=\omega_{0} \Delta \omega \\
\Delta \dot{\omega}=\omega_{0} \Delta \omega \\
\Delta \dot{\omega}=\frac{T_{m}-T_{e}}{2 H}, \\
T_{e}=K_{2} \Delta \varphi_{f d} \\
\Delta \varphi_{f d}=\left(\Delta E_{f d}-K_{4} \Delta \delta\right) \frac{K_{3}}{1+S T_{R}}, \\
\Delta E_{f d}=\left(-V_{t}+U_{i}\right) K_{A} \\
V_{t}=\left(K_{5} \Delta \delta+K_{6} \Delta \varphi_{f d}\right) \frac{1}{1+S T_{R}}
\end{gathered}
$$

where are

$\delta \quad$ : angle between quadrature axis and infinite bus

$\omega_{0} \quad: \quad 2 \pi \dot{f}, f=60 \mathrm{~Hz}$.

$\omega \quad$ : rotor speed.

$T_{m} \quad$ : mechanical torque.

$T_{e} \quad$ : electrical torque.

$H \quad$ : inertia coefficient.

$E_{f, d} \quad$ : generator field voltage.

$K_{A}, T_{R}$ : exciter gain and time constant.

$V_{\text {ref }} \quad$ : reference voltage.

$V_{t} \quad$ : terminal voltage.

$K_{1}$ to $K_{6}$ are constant parameters that they are dependent on the machine parameters and the operating conditions. Generally $K_{1}, K_{2}, K_{3}$ and $K_{6}$ are positive. $K_{4}$ is mostly positive except for cases where line impedance is high. $K_{5}$ is positive for low to medium line impedances and low to medium loading. $K_{5}$ is usually negative for medium to high line impedances and heavy loading. The $K$-parameters of the machine expressed in terms of $P$ and $Q$ can be discussed as [16] by equations (2) and (3).

$$
\begin{aligned}
& K_{1}=R_{3} \frac{P^{2}}{P^{2}+(Q+R)^{2}}+Q+R_{1}, \\
& K_{2}=R_{4} \frac{P}{\sqrt{P^{2}+\left(Q+R_{1}\right)^{2}}}, \\
& K_{3}=\frac{x_{d}^{\prime}+x_{e}}{x_{d}+x_{e}} \text {, } \\
& K_{4}=R_{5} \frac{P}{\sqrt{P^{2}+\left(Q+R_{1}\right)^{2}}}, \\
& K_{5}=R_{4} x_{e} \frac{P}{V_{t}^{2}+Q x_{e}}\left[R_{6} \frac{Q+R_{1}}{P^{2}+\left(Q+R_{1}\right)^{2}}\right] \text {, } \\
& K_{6}=R_{7} \frac{\sqrt{P^{2}+\left(Q+R_{1}\right)^{2}}}{V_{t}^{2}+Q x_{e}}\left[x_{e}+\frac{R_{1} x_{q}\left(Q+R_{1}\right)}{P^{2}+\left(Q+R_{1}\right)^{2}}\right] \text {. } \\
& R_{1}=\frac{V_{t}^{2}}{x_{e}+x_{1}}, \quad R_{2}=K_{3}, \\
& R_{3}=R_{1} \frac{x_{q}-x_{d}^{\prime}}{x_{e}+x_{d}^{\prime}}, \quad R_{4}=\frac{V_{t}}{x_{e}+x_{d}^{\prime}}, \\
& R_{5}=\frac{x_{d}-x_{d}^{\prime}}{x_{e}+x_{d}^{\prime}}, \quad R_{6}=R_{1} \frac{x_{q}\left(x_{q}-x_{d}^{\prime}\right)}{x_{e}+x_{q}}, \\
& R_{7}=\frac{x_{e}}{x_{e}+x_{d}^{\prime}}
\end{aligned}
$$

where are 
$K_{1}, \ldots, K_{6}$ : the $K$-parameters of the synchronous generator.

$X_{q} \quad$ : quadrature-axis synchronous reactance.

$X_{d}^{\prime}, x_{d} \quad$ : generator direct-axis transient reactance, and direct-axis synchronous reactance respectively.

$x_{e} \quad$ : line reactance.

$P, Q \quad$ : real and reactive power loading respectively.

\subsection{PSS Structure}

PSS is used to compensate the phase lag between the exciter input and the machine electrical torque by producing a proper torque on the rotor of the machine. A widely used conventional lead-lag PSS, is considered in this study. In this structure, Power System Stabilizer (PSS) consists of a gain block with gain $k_{w}$, a signal washout block and two-stage phase compensation blocks. This structure is shown in Fig. 2, and follows from the equation

$$
U_{i}=k_{w} \frac{s T_{w}}{1+s T_{w}} \frac{1+s T_{p 1}}{1+s T_{p 2}} \frac{1+s T_{p 3}}{1+s T_{p 4}} \Delta \omega .
$$

As is shown in Fig. 2, the stabilizer includes a high pass filter where $k_{w}$ and $T_{p 1}$, and $T_{p 3}$ are Power System Stabilizer adjustable parameters, and $T_{w}, T_{p 2}$, and $T Y_{p 4}$ are considered constants $T_{p 2}=T_{p 4}=0.05, T_{w}=10$.

\section{OVERVIWE OF WATER CYCLE ALGORITHM}

Water cycle algorithm (WCA) is a novel optimization method for solving constrained engineering optimization problems that proposed in 2012 [17]. In this algorithm sea is best value and stream or river as initial population, adjoined to sea (best value) at last.

Water Cycle Algorithm (WCA) has ability to find maximum or minimum value of function with high speed and accuracy, so in this paper, this algorithm is applied to determine the optimum Power System Stabilizer (PSS) parameters in which power system oscillation is minimum. In fact, problem is to design the parameters of the power system stabilizer.

In a problem with $N_{v a r}$ variables, each raindrop is a $1 \times N_{\text {var }}$ array and each array is a solution for problem. These arrays are put in a matrix

$$
\begin{gathered}
\text { Raindrop }_{i}=X_{i}=\left[x_{1}, x_{2}, \ldots, x_{N v a r}\right], \\
\text { Population raindrops }=\left[\begin{array}{c}
\text { raindrop }_{1} \\
\ldots \\
\text { raindrop }_{i} \\
\ldots \\
\text { raindrop }_{N p o p}
\end{array}\right]
\end{gathered}
$$

so $N_{\text {pop }}$ is number of raindrops and population raindrops are initial population. Then cost of each raindrop is calculated by cost function.

\subsection{Stream (or river) flow to river (or sea)}

All streams and rivers join to the sea at last by expressions

$$
\begin{aligned}
& \text { Position }_{\text {stream }}^{\text {new }}=\text { Position }_{\text {stream }}+ \\
& \text { rand } \times C \times\left(\text { Position }_{\text {river }}-\text { Position }_{\text {stream }}\right),
\end{aligned}
$$

$$
\begin{aligned}
\text { Position }_{\text {river }}^{\text {new }} & =\text { Position }_{\text {river }}+ \\
& \text { rand } \times C \times\left(\text { Positin }_{\text {sea }}-\text { Position }_{\text {river }}\right)
\end{aligned}
$$

where $C$ is a number between 1 and 2 . rand is a uniformly distributed random number between 0 and 1 . If the solution is given by a stream is better than its connecting river, the positions of river and stream are exchanged ( $i e$ stream becomes river and river becomes stream). Such exchange can similarly happen for rivers and sea.

\subsection{Evaporation and Raining}

In order to avoid getting trapped in local optima evaporation and raining process is proposed [17]. This is specific prominence for Water Cycle Algorithm (WCA) to comparison other optimization algorithms. Evaporation process end if

$$
\mid \text { Position }_{\text {sea }}-\text { Position }_{\text {river }} \mid<\mathrm{d}_{\text {max }} \text {, }
$$

$\mathrm{d}_{\max }$ is a number near to 0 . The value of $\mathrm{d}_{\max }$ automatically decreases as

$$
\mathrm{d}_{\max }^{\text {new }}=\mathrm{d}_{\max }-\left(\mathrm{d}_{\max } / \text { maxiteration }\right) .
$$

After evaporation process, raining process begins.

In the raining process, the new raindrops are flowing to streams in the different locations. Eq (10) is used for new location of streams.

$$
\operatorname{Position}_{\text {stream }}^{\text {new }}=\operatorname{Position}_{\text {sea }}+\sqrt{U} \times \operatorname{randn}\left(1, N_{\text {var }}\right)
$$

where $U$ determines rate of search near to the sea. randn is the normally distributed random number.

\subsection{End of loop}

In this paper, maximum number of iterations (maxiteration) is used as a criterion for end of main loop which at the end loop, the optimal parameters are received.

Water Cycle Algorithm (WCA) parameters has been brought in Tab. 1.

Table 1. Water cycle algorithm (WCA) parameters

\begin{tabular}{ll}
\hline Parameters & Value \\
\hline$N_{\text {var }}$ & 3 \\
$N_{\text {pop }}$ & 30 \\
$C$ & 2 \\
$U$ & 0.1 \\
$\mathrm{~d}_{\max }$ & 0.001 \\
Maxiteration & 50 \\
\hline
\end{tabular}


Table 2. Water cycle algorithm (WCA) outputs

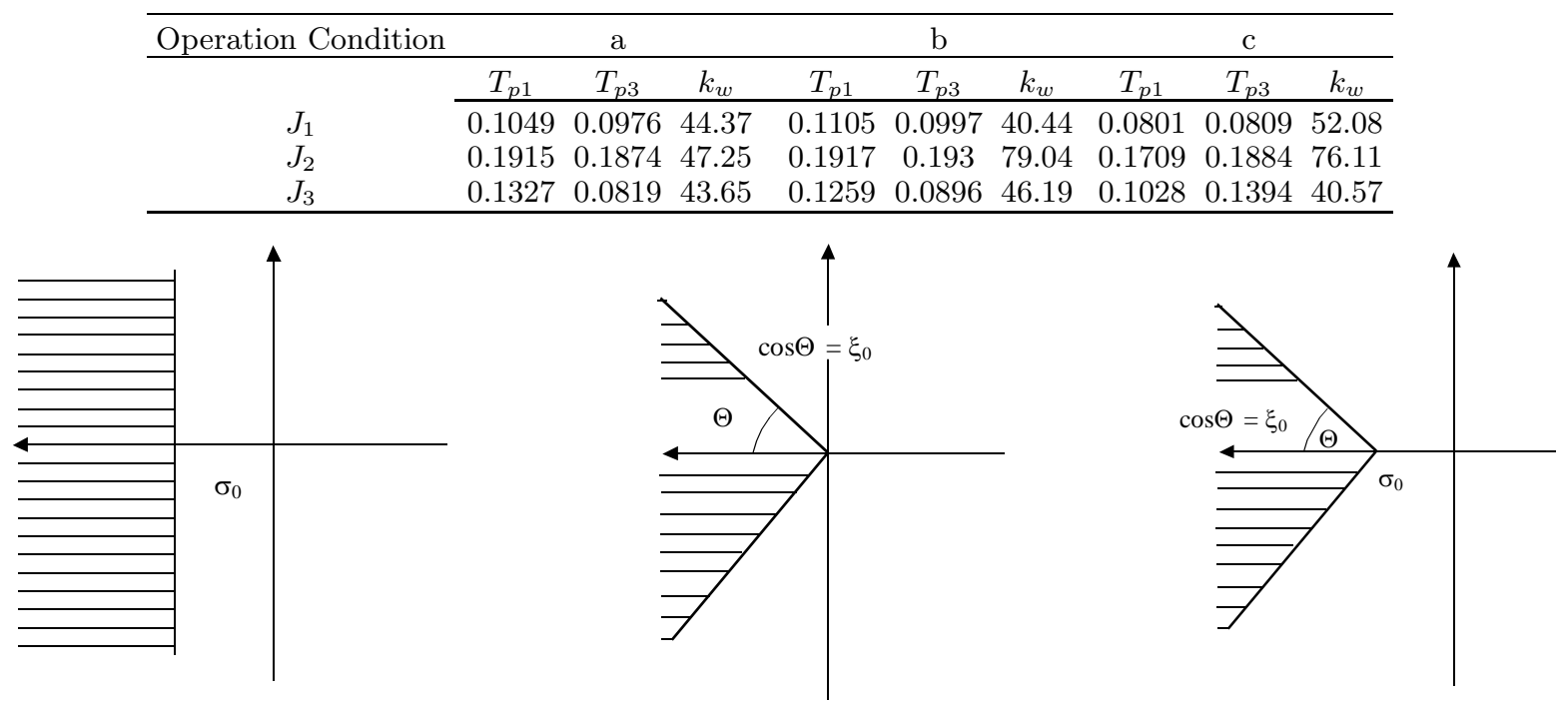

Fig. 3. $J_{1}$ operation region

Fig. 4. $J_{2}$ operation region

Fig. 5. $J_{3}$ operation region

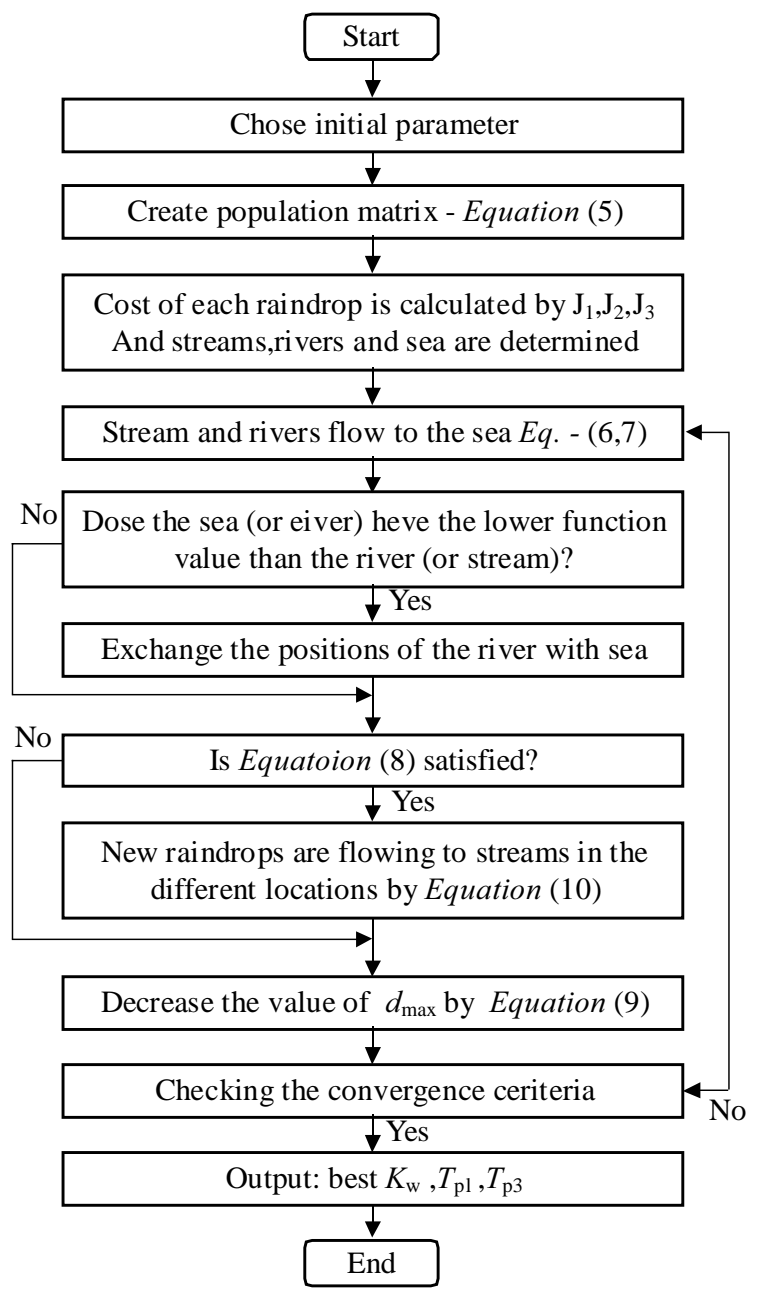

Fig. 6. Flowchart of proposed algorithm

\section{OPTIMIZATION PROBLEM FORMULATION}

In this present study, washout time $T_{w}, T_{p 2}$ and $T_{p 4}$ are considered constants, and $k_{w}, T_{p 1}$, and $T_{p 3}$ should be determined. Consider, that the aim of main objective is to design Power System Stabilizer (PSS) which minimizes the power system oscillations in reasonable computation time.

In this paper, we consider three subsidiary objectives to determine the various parameters of Power System Stabilizer (PSS). The first objective is to determine the relative stability of the system. So the eigenvalues of system in order to access desirable level of damping ratio $\left(\sigma_{0}\right)$ should be transferred to the left of $s=\sigma_{0}$, that is demonstrated in Fig. 3:

$$
J_{1}=\sum_{\sigma_{i}>\sigma_{0}}\left(\sigma_{i}-\sigma_{0}\right)^{2}
$$

where $\sigma_{i}$ is damping ratio, $\sigma_{0}=1.5$ is considered, and $J_{1}$ is the first subsidiary objective function.

The second subsidiary objective is to limit the overshoot of transferred eigenvalues to the specific area connector as Fig. 4. This causes to access desirable damping coefficient $\left(\xi_{0}\right)$ :

$$
J_{2}=\sum_{\xi_{i}>\xi_{0}}\left(\xi_{i}-\xi_{0}\right)^{2}
$$

where $\xi_{i}$ is damping coefficient, $\xi_{0}=0.2$ is considered, and $J_{2}$ is the second subsidiary objective function.

The third subsidiary objective is conflation two prior subsidiary objectives and can be expressed by the Figure 5 and following form

$$
J_{3}=\alpha J_{1}+\beta J_{2}
$$

where $\alpha=1$ and $\beta=10$ are considered, and $J_{1}, J_{2}$ and $J_{3}$ are first, second, and third subsidiary objective functions, respectively. Minimizing $J_{i}(i=1,2,3)$ subject to

$$
\begin{aligned}
k_{w, \min } & <k_{w}<k_{w, \max }, \\
T_{P 1, \min } & <T_{P 1}<T_{P 1, \max }, \\
T_{P 3, \min } & <T_{P 3}<T_{P 3, \max } .
\end{aligned}
$$


Table 3. Details of these three operation conditions

\begin{tabular}{ccccccc}
\hline Operation Condition & \multicolumn{2}{c}{$\mathrm{a}$} & $\mathrm{b}$ & \multicolumn{2}{c}{$\mathrm{c}$} \\
\hline & Overshoot & $\begin{array}{c}\text { Settling } \\
\text { time(s) }\end{array}$ & Overshoot & $\begin{array}{c}\text { Settling } \\
\text { time(s) }\end{array}$ & Overshoot & $\begin{array}{c}\text { Settling } \\
\text { time(s) }\end{array}$ \\
\cline { 2 - 7 }$J_{1}$ & $2.55 \times 10^{-3}$ & 3.51 & $1.36 \times 10^{-3}$ & 3.46 & $1.32 \times 10^{-3}$ & 3.31 \\
$J_{2}$ & $0.89 \times 10^{-3}$ & 4.16 & $0.34 \times 10^{-3}$ & 4.17 & $0.233 \times 10^{-3}$ & 4.52 \\
$J_{3}$ & $1.73 \times 10^{-3}$ & 3.60 & $0.41 \times 10^{-3}$ & 3.68 & $0.301 \times 10^{-3}$ & 3.48 \\
\hline
\end{tabular}

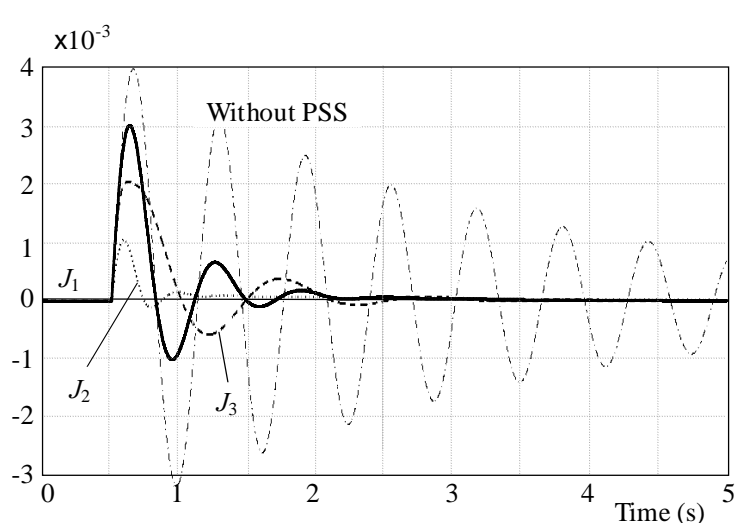

Fig. 7. Objective functions $J_{i}(i=1,2,3)$ under light loading

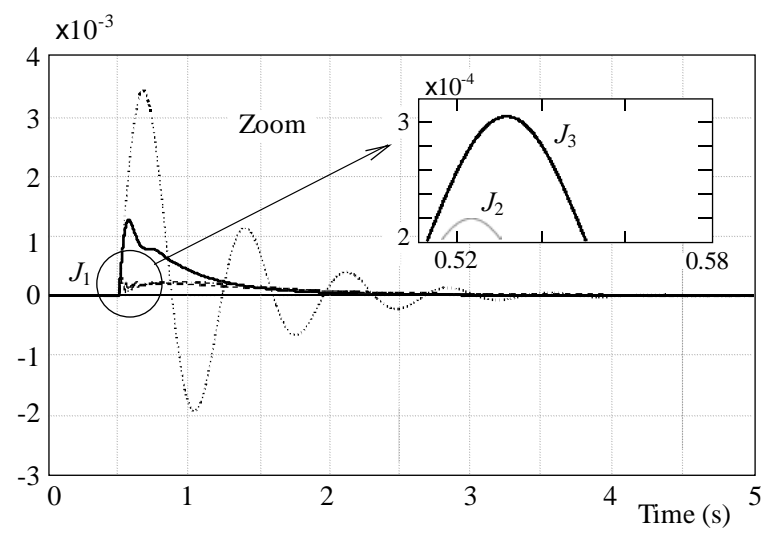

Fig. 9. Objective functions $J_{i}(i=1,2,3)$ under heaving loading

The flowchart of Water Cycle Algorithm (WCA) with considering above objectives for detecting optimum Power System Stabilizer (PSS) parameters is shown in Fig. 6.

\section{SIMULATION RESULTS}

In order to assessment and detecting the efficiency and accuracy of the proposed controller, a Heffron-Phillip model is developed for Single machine infinite bus system using MATLAB/SIMULINK. This system is simulated under different operating conditions and severe disturbances. The severe disturbance is applied at $t=0.5 \mathrm{~s}$, and its amplitude is $0.2 \mathrm{pu}$. Three operation conditions are considered:

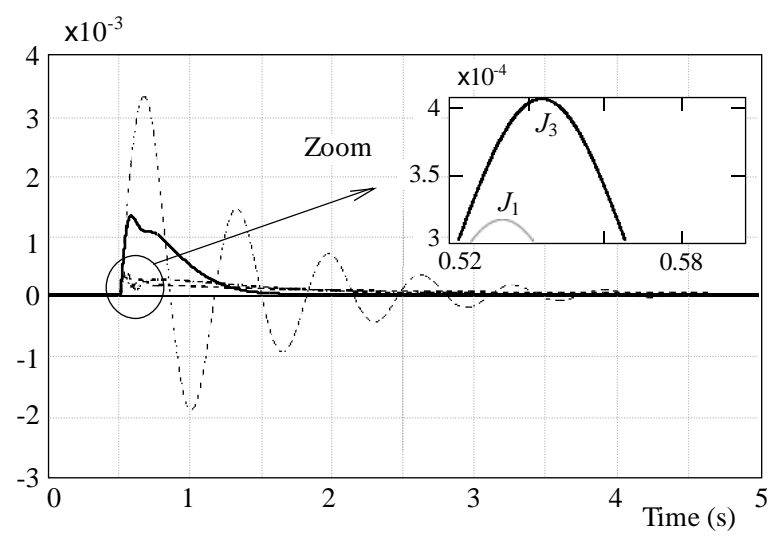

Fig. 8. Objective functions $J_{i}(i=1,2,3)$ under nominal loading

a) Light loading

b) Nominal loading

c) Heavy loading

By applying Water Cycle Algorithm (WCA) according Tab. 1 to this system, the Power System Stabilizer (PSS) parameters in three operation conditions are received as Tab. 2.

Figure 7 shows $J_{i}(i=1,2,3)$ which are objective functions under light loading. As is shown in this figure for light loading the $J_{2}$ has the least overshoot among other function, but $J_{1}$ has the least settling time. For nominal loading, as is shown in Fig. $8, J_{2}$ has the least overshoot among other function, but $J_{1}$ has the least settling time. This condition is confirmed for heaving loading which is shown in Fig. 9. Details of these three operation conditions is summarized in Tab. 3 .

To showing the efficiency and accuracy of this proposed controller, its results in three conditions (light, nominal and heaving loading) are compared while the PSS is lack in the system, and are shown in Figs. 7,8,9. This demonstrates that the overshoot, undershoot, settling time and speed deviations of machine are greatly reduced by applying the proposed WCA based tuned PSS.

To explain performance of the proposed method, one performance index Figure of Demerit (FD) based on the system operation is expressed as [18]

$$
F D=(1000 \times O S)^{2}+(1000 \times U S)^{2}+T_{S}^{2}
$$

where Overshoot $(O S)$, Undershoot $(U S)$ and settling time of rotor angle deviation in machine $\left(T_{S}\right)$ are used 
to evaluation the $F D$. So that, the best system response is reached when the $F D$ index value is the lowest. Numerical results confirm with considering $F D$ index, the objective function $J_{3}$ has the best operation as shown in Tab. 4.

Table 4. Numerical result of $F D$ index

\begin{tabular}{|c|c|c|c|}
\hline $\begin{array}{l}\text { Operation } \\
\text { Condition }\end{array}$ & $\mathrm{a}$ & $\mathrm{b}$ & $\mathrm{c}$ \\
\hline & \multicolumn{3}{|c|}{$F D$ index value } \\
\hline$J_{1}$ & 19.54 & 13.82 & 12.72 \\
\hline$J_{2}$ & 18.10 & 17.5 & 20.48 \\
\hline$J_{3}$ & 16.18 & 13.71 & 12.20 \\
\hline
\end{tabular}

\section{CONCLUSION}

In this paper, the Water Cycle Algorithm (WCA) has been proposed to optimally turn the PSS parameters and by reduction of oscillations, improve power system stability. To optimize the PSS parameters, three objective functions have been considered which finally caused to reduce overshoot and settling times in different operation conditions. The simulation results shown that the first objective function $J_{1}$, reduced settling time, second objective function $J_{2}$ reduced overshoot and third objective function $J_{3}$ that is conflation of $J_{1}$ and $J_{2}$ properly improve overshoot and settling time. Using $F D$ index in proposed WCA based tuned PSS revealed that, the overshoot, undershoot, settling time and speed deviations of the machine are greatly reduced under severe disturbance conditions. Also comparing between these results and results of without PSS condition, confirm that WCA is successful in detecting the optimal PSS parameters.

\section{REFERENCES}

[1] LARSEn, E.-SWANn, D.: IEEE Trans. Power App. 100 (1981), 3017-3046.

[2] SIDHARTHA PANDA-PADHY, N. P.: Robust Power System Stabilizer Design using Particle Swarm Optimization Technique, International Journal of Electrical Systems Science and Engineering 1 No. 1.

[3] USMAN, J.-MUSTAFA, M. W.-ALIYA, G. : Design of AVR and PSS for Power System Stability based on Iteration Particle Swarm Optimization, International Journal of Engineering and Innovative Technology (IJEIT) 2 No. 6 (Dec 2012).

[4] ABIDO, M. A.: Optimal Design of Power System Stabilizers Using Particle Swarm Optimization, IEEE Transactions on Energy Conversion 17 No. 3 (2002).

[5] SOliman, H. M.-BAYOUMI, E. H. E.-HASSAN, M. F.: PSO-Based Power System StabIlizer for Minimal Overshoot and Control ConstraintsJOUR Journal of Electrical Engineering.
[6] LEE, S.: Optimal Decentralised Design for Output-Feedback Power System Stabilizers, IEE Proc. Generation Transmission and Distribution 152 No. 4 (2005), 494-502.

[7] FRAile-ARDAnUY, J.-ZUfiRIA, P. J.: Design and Comparison of Adaptive Power System Stabilizers based on Neural Fuzzy Networks and Genetic Algorithms, J. Neurocomputing 70 (2007), 2902-2912.

[8] SEGAL, R.-SHARMA, A.-KOTHARI, M. L.: A Self Tuning Power System Stabilizer based on Artificial Neural Network, Electric. Power Energy Systems 26 (2004), 423-430.

[9] SAOUDI, K.-HARMAS, M. N. : Enhanced Design of an Indirect Adaptive Fuzzy Sliding Mode Power System Stabilizer for Multi-Machine Power Systems, International journal of Electrical Power and Energy Systems 54 (2014), 425-431.

[10] AlKhatiB, H.-DUVEAU, J.: Dynamic Genetic Algorithms for Robust Design of Multimachine Power System Stabilizers, International journal of Electrical Power and Energy Systems 45 (2013), 242-251.

[11] ABDEL-MAGID, Y. L.-ABIDO, M. A. : Optimal Multi Objective Design of Robust Power System Stabilizers using Genetic Algorithms, IEEE Trans. on Power Systems 18 No. 3 (2003), $1125-1132$.

[12] ABIDO, M. A.: Robust Design of Multimachine Power System Stabilizers using Simulated Annealingjour IEEE Trans. on Energy Conversion.

13] REKHA, S. S.: Multi Machine Power System Stabilizer Design Using Particle Swarm Optimization Technique, International Journal of Engineering Inventions 2 No. 6 (Apr 2013), 102-106.

14] DUBEY, M.-DUBEY, A.-ShrivastavA, A.: PSO Based Power System Stabilizer To Improve Power System Stability, 2nd International Conference on Mechanical, Electronics and Mechatronics Engineering; 17-18 2013 June London.

[15] SedighizadeH, M.-Minooie, B.-SARVI, M.: Power System Stability Enhancement using a NSPSO Designed UPFC Damping Controller, International Journal on Technical and Physical Problems of Engineering (IJTPE) 14 No. 5 (2013), 1-8.

[16] SHAYEGHI, H.-SHAYANFAR, H. A.-GHASEMI, A. : Artificial Bee Colony Based Power System Stabilizer Design for a Turbo-Generator in a Single-Machine Power System.

[17] ESKANDARI, H.-SADOLLAH, A.-BAHREININEJAD, A. -HAMDI, M. : Water Cycle Algorithm-A Novel Metaheuristic Optimization Method for Solving Constrained Engineering Optimization Problems, J. Computer and Structures 110 (2012), 151-166.

[18] SHAYEGHI, H.-JALILI, A.-SHAYANFAR, H. A.: MultiStage Fuzzy Load Frequency Control using PSO, Energy Conversion and Management 49 (2008), 2570-2580.

Received 11 December 2013

Navid Ghaffarzadeh is an assistant professor of electrical engineering at Imam Khomeini International University. He received $\mathrm{MS}$ and $\mathrm{PhD}$ degrees in electrical engineering from the Amirkabir University of Technology (graduated with First Class Honors), Tehran, Iran, and the Iran University of Science and Technology (graduated with First Class Honors), Tehran, Iran, respectively. His research interests include power systems protection and control, transient in power systems and power quality. He is the author of three books in the field of power systems. He is also the author and the coauthor of 62 technical papers. 